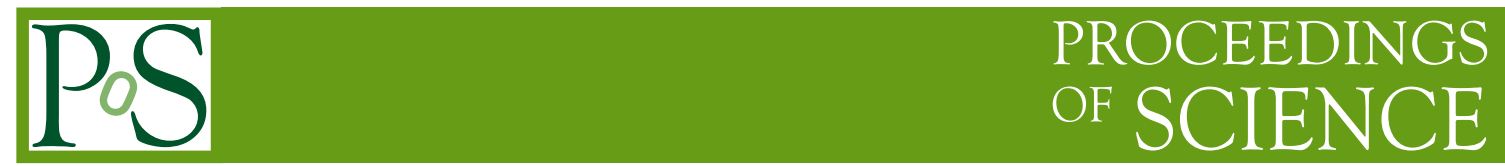

\title{
Search for new heavy gauge bosons at CMS
}

\author{
Claudia-Elisabeth Wulz ${ }^{* \dagger}$ \\ Institute of High Energy Physics, Vienna, Austria \\ E-mail: claudia.wulz@cern.ch
}

\begin{abstract}
We present results of searches for new heavy gauge bosons decaying to dilepton, diphoton, lepton and missing transverse energy, $W Z$, and $Z Z$ final states. The full sample of $5 \mathrm{fb}^{-1}$ of pp collisions at $7 \mathrm{TeV}$ collected with CMS in 2011 as well as the new collisions recorded at $8 \mathrm{TeV}$ in 2012 have been analyzed.
\end{abstract}

36th International Conference on High Energy Physics

4-11 July 2012

Melbourne, Australia

\footnotetext{
*Speaker.

On behalf of the CMS Collaboration.
} 
The Standard Model (SM) has so far quite successfully described the strong and electroweak interactions of fundamental particles. However, it does not explain phenomena such as gravity, dark matter or dark energy. Furthermore, it does not achieve unification of all forces. To overcome these and other shortcomings, numerous extensions have been proposed. The Standard Model $S U(3) \otimes S U(2)_{L} \otimes U(1)_{Y}$ gauge group can for example be extended by an extra $U(1)$ group, giving rise to a neutral heavy vector boson $Z^{\prime}$, or an extra $S U(2)$ group, giving rise to a charged heavy vector boson $W^{\prime}$. Some models that have been developed with these assumptions are:

- Sequential Standard Model (SSM) [1]

The new bosons $W^{\prime}$ and $Z^{\prime}$ have similar couplings as the $W$ and $Z$ in the SM. This model has been used as a reference model in many collider physics analyses.

- Left-right symmetric models (LR) [2]

These models contain an extra $S U(2)_{R}$ gauge group.

- $E_{6}$ models [3]

These models are inspired by Superstrings. They contain the group $E_{6}$, which is broken down as $E_{6} \rightarrow S O(10) \otimes U(1)_{\psi} \rightarrow S U(5) \otimes U(1)_{\chi} \otimes U(1)_{\psi}$, with $S U(5)$ broken to the SM gauge group.

There are also more complicated scenarios, predicting a tower of new gauge bosons $\left(W^{n}, Z^{n}\right.$, or gravitons $G^{n}$ ), such as extra dimensions [4, 5], composite Higgs or technicolor models.

CMS [6] has searched for new heavy gauge bosons in several decay channels.

\section{1. $Z^{\prime}$ decaying to dileptons}

Many $Z^{\prime}$ models predict narrow resonances decaying to dileptons. A shape analysis of the dielectron and dimuon mass spectra has been performed, in order to be robust against uncertainties in the absolute background level coming from Drell-Yan $Z / \gamma^{*}$ production, either directly or through $\tau \bar{\tau}, t \bar{t}$, or from multijets with at least one jet misreconstructed as a lepton. From the $2012 \sqrt{s}=$ $8 \mathrm{TeV}$ proton-proton data sample dileptons have been selected using transverse momentum $\left(\mathrm{p}_{T}\right)$ cuts of $35 \mathrm{GeV}$ and $45 \mathrm{GeV}$ for electrons and muons, respectively. Isolation criteria have been applied as well. Fig. 1 shows the dimuon spectrum obtained with $4.1 \mathrm{fb}^{-1}$ and the ratio $R_{\sigma}$ of the production cross section times branching fraction for a $Z^{\prime}$ in the SSM and a $Z_{\psi}^{\prime}$ to that for the $Z$ boson. The ratio plot includes also data taken at $7 \mathrm{TeV}$ in 2011. Since no excess over the SM background has been found, mass limits have been set for a new neutral gauge boson. Using 2011 and 2012 data, the SSM $Z^{\prime}$ can be excluded below $2590 \mathrm{GeV}$, and the $Z_{\psi}^{\prime}$ below $2260 \mathrm{GeV}$, at $95 \%$ confidence level [7].

The $Z^{\prime}$ decay channel into $\tau$ 's has also been studied [8]. Usually generation-independent couplings to fermions are assumed, but there are non-universal scenarios in which the $Z^{\prime}$ couples preferentially to third-generation fermions [9]. Four $\tau$ final states have been studied with the $7 \mathrm{TeV}$ data $-\tau_{e} \tau_{\mu}, \tau_{e} \tau_{h}, \tau_{\mu} \tau_{h}$ and $\tau_{h} \tau_{h}$, where $\tau_{e}$ and $\tau_{\mu}$ are the electron and muon decay modes, and $\tau_{h}$ is the hadronic decay mode into one, three or five charged mesons, which can be accompanied by neutral pions. The presence of the $\tau$-neutrino does not allow to reconstruct the mass of the 

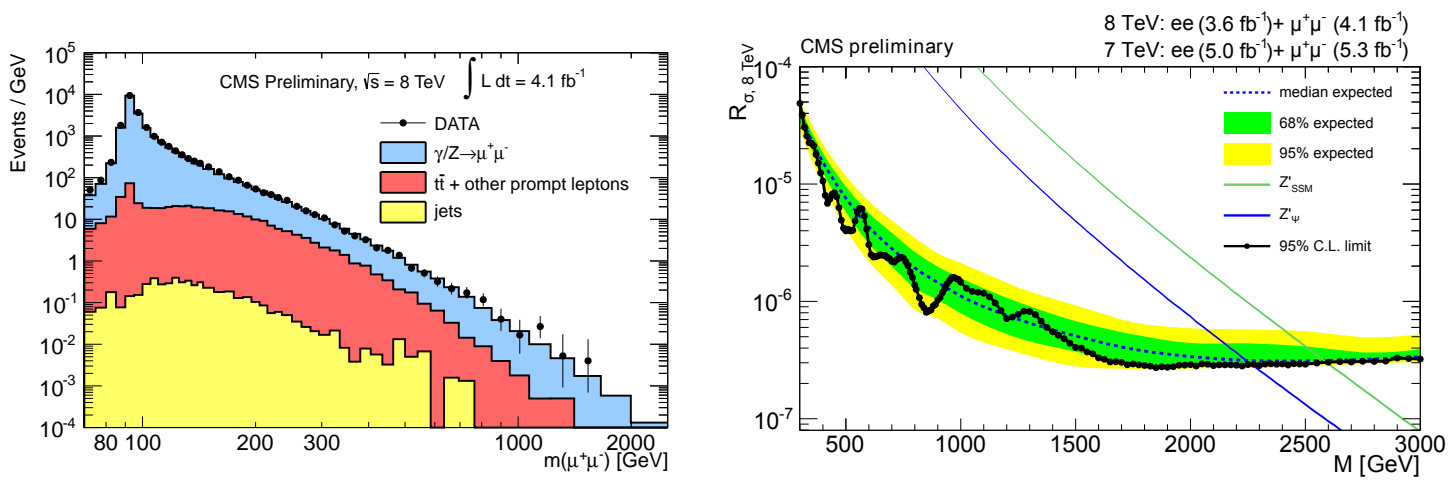

Figure 1: (a) Invariant mass spectrum of dimuons, (b) Ratio of production cross section times branching fraction for $Z^{\prime}$ and $Z$.

$\tau \tau$-system. The event selection required two isolated $\tau$ candidates with $\mathrm{p}_{T}$ between 15 and $35 \mathrm{GeV}$ and within a pseudorapidity interval $|\eta|<2$.1. In addition, any b-jets were vetoed. Backgrounds coming from $Z \rightarrow \tau \tau, W+$ jets, $t \bar{t}$, vector boson pairs, and QCD have been estimated from data where possible. In Fig. 2 a the effective visible mass for the $\tau_{h} \tau_{h}$ channel with background estimations is plotted. Fig. $2 b$ shows the $Z$ 'production cross section times the $\tau \tau$ branching fraction. In the absence of a signal, $Z_{S S M}^{\prime}$ and $Z_{\psi}^{\prime}$ resonances were excluded for masses below 1.4 and $1.1 \mathrm{TeV}$ at $95 \%$ confidence level, respectively.
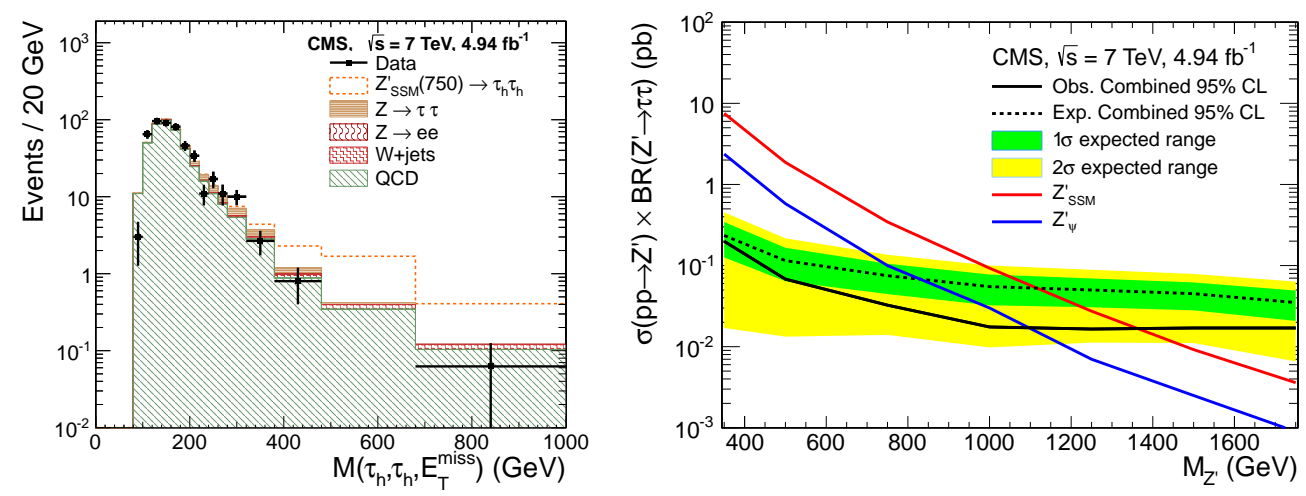

Figure 2: (a) Effective visible mass for the $\tau_{h} \tau_{h}$ decay channel, (b) Production cross section times branching fraction for $Z_{S S M}^{\prime}$ and $Z_{\psi}^{\prime}$.

\section{2. $\mathrm{W}^{\prime}$ decaying to leptons and neutrinos}

As for the $Z^{\prime}$, a SSM $W^{\prime}$ has been studied, where in contrast to the SM $W$ the decay $W^{\prime} \rightarrow t b$ is allowed. The event selection required an isolated lepton back-to-back with balanced missing transverse energy $\left(\mathrm{E}_{T}^{\text {miss }}\right)$. Backgrounds include events from $W \rightarrow \ell v, \mathrm{QCD}, t \bar{t}$ and single top, Drell-Yan and vector boson pairs. They have been estimated from data. No interference between $W^{\prime}$ and $W$ has been assumed. Both for the electron and the muon channels no significant excess is observed. The data have also been interpreted in the context of a Universal Extra Dimension (UED) framework, which assumes one additional compact dimension $R$. In a split-UED model [10] the parameter space is defined by the parameters $1 / R$ and $\mu$, where $\mu$ is the bulk mass parameter 
of the fermion field in five dimensions. Fig. 3a shows the excluded mass region in the $[1 / R, \mu]$ parameter space, derived from all available data. Fig.3b shows the observed and expected limits for the production cross section times branching fraction for a $W_{S S M}^{\prime}$ and theoretical expectations for a Kaluza-Klein $W_{K K}^{n=2}$ for values of $\mu=10 \mathrm{TeV}$ and $\mu=0.05 \mathrm{TeV}$. This is the lowest state that can couple to SM fermions. Combining the electron and muon channels, one obtains a lower $W^{\prime}$ mass limit of $2.9 \mathrm{TeV}$ from the $5 \mathrm{fb}^{-1}$ of $7 \mathrm{TeV}$ data and the $3.7 \mathrm{fb}^{-1}$ of the $8 \mathrm{TeV}$ sample.
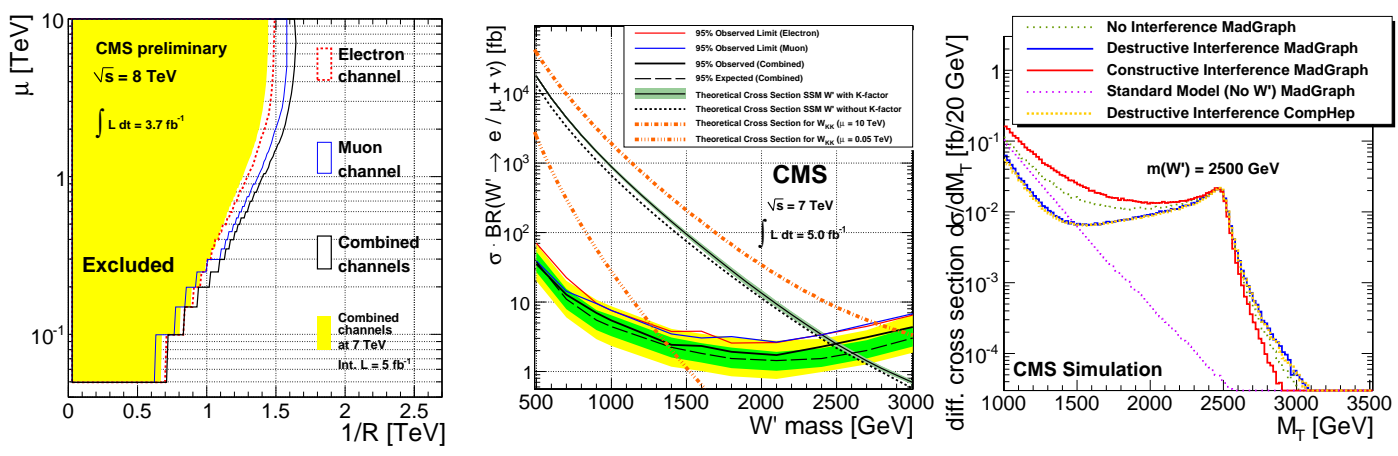

Figure 3: (a) Excluded region in split-UED parameter space, (b) Production cross section times branching fraction for $W_{S S M}^{\prime}$ and $W_{K K}^{n=2}$, (c) Simulation of interference effects.

A left-handed $W^{\prime}$ can interfere with the $W$. With the $7 \mathrm{TeV}$ data studies including interference have also be performed [11]. The effects of interference can be seen in Fig. 3c, which shows simulations with MadGraph and CompHep. The mass limits range from $2.63 \mathrm{TeV}$ for constructive interference to $2.43 \mathrm{TeV}$ for destructive interference. Using the same data and selection, an interpretation in terms of a right-handed $W_{R}^{\prime}$ yields a limit of $2.5 \mathrm{TeV}$.

\section{3. $\mathrm{W}^{\prime}$ decaying to tb or td}

In many models the third generation is coupled more strongly to the $W$ than the other two generations. The decays to leptons are suppressed if the right-handed neutrino is heavier than the $W^{\prime}$. The decay chain $W \rightarrow t b \rightarrow W b b \rightarrow \ell v b b$ has been studied. The event selection was based on isolated electrons (muons) with $\mathrm{p}_{T}>35(32) \mathrm{GeV}$, two jets with $\mathrm{E}_{T}^{\text {jet } 1 \text { (jet2) }}>100(40) \mathrm{GeV}$, and at least one b-tagged jet. Backgrounds are $t \bar{t}$ and single top, $W+$ jets, $Z / \gamma^{*}+$ jets, $\mathrm{QCD}$ and vector boson pairs. A new boosted decision tree (BDT) analysis with about 50 variables, including object and event kinematics, top reconstruction and angular correlations, has been used to facilitate the discrimination of the signal from background for a $W_{R}^{\prime}$ boson. The mass sensitivity is increased by about $200 \mathrm{GeV}$ compared to a previously used invariant mass method, as shown in Fig. 4a. For the first time it has also been possible to derive limits on coupling strengths. The most general model-independent lowest-order Lagrangian for a $W^{\prime}$ coupling to SM fermions is given by:

$$
\mathscr{L}=\frac{V_{f_{i} f_{j}}}{2 \sqrt{2}} g_{w} \bar{f}_{i} \gamma_{\mu}\left(a_{f_{i} f_{j}}^{R}\left(1+\gamma^{5}\right)+a_{f_{i} f_{j}}^{L}\left(1-\gamma^{5}\right)\right) W^{\prime \mu} f_{j}+\text { h.c. }
$$

For the left- and right-handed couplings $a_{f_{i} f_{j}}^{L, R}$ it was assumed that $a_{u d}^{L, R}=a_{c s}^{L, R}=a_{t b}^{L, R}=a^{L, R}$. The corresponding $a^{L}-a^{R}$ contours of the $W^{\prime}$ masses at which the observed $95 \%$ cross section upper limit equals the predicted cross section are plotted in Fig. $4 \mathrm{~b}$. 

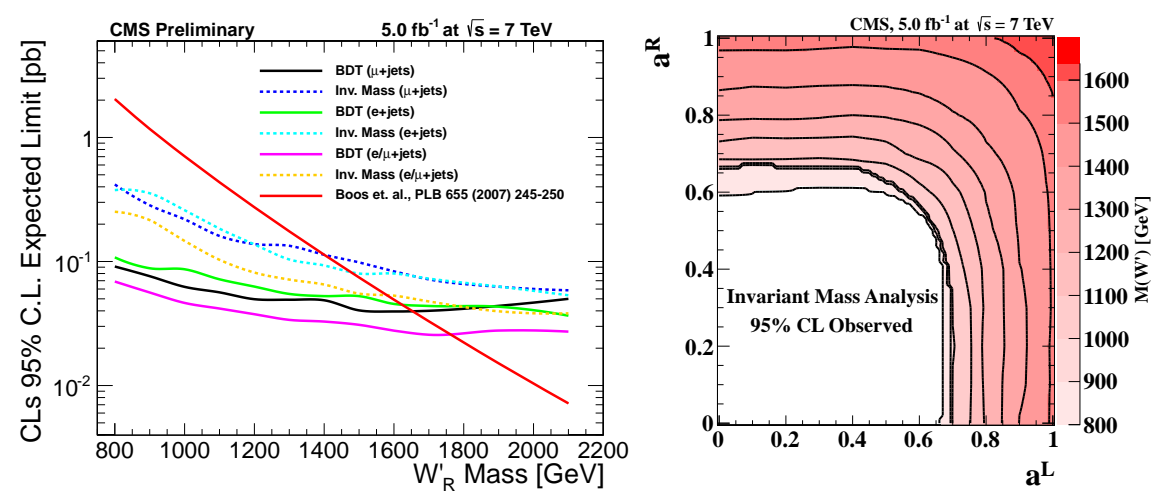

Figure 4: (a) Sensitivity comparison for the BDT and the invariant mass methods, (b) Contours.

A search for $W^{\prime}$ produced in association with a top quark has also been performed in the $t t d$ final state, requiring semileptonic $t$ decays and an additional jet. One of the jets was required to be coming from a $b$. The search was also motivated by Tevatron measurements of the $t \bar{t}$ forwardbackward asymmetry, which show a possible deviation from the SM in the high-end tail of the distribution and which could be explained by a $W^{\prime}$ in the 200 to $600 \mathrm{GeV}$ mass range. At the LHC the ratio of $W^{\prime-}$ to $W^{\prime+}$ production is $85 \% / 15 \%$. This feature is used to aid in the reconstruction of the $W^{\prime}$. The difference of yields for the $t^{-}+d$ and $t^{+}+d$ mass distributions is shown in Fig. 5a. The analysed $7 \mathrm{TeV}$ data show no asymmetry, whereas a $W^{\prime}$ of $600 \mathrm{GeV}$ mass would lead to sizeable fluctuations. Combining electron and muon channels, limits were derived for the $W^{\prime}$ cross section at $95 \%$ confidence level as shown in Fig. $5 \mathrm{~b}$. The $W^{\prime}$ is excluded for masses below $839 \mathrm{GeV}$ [12].
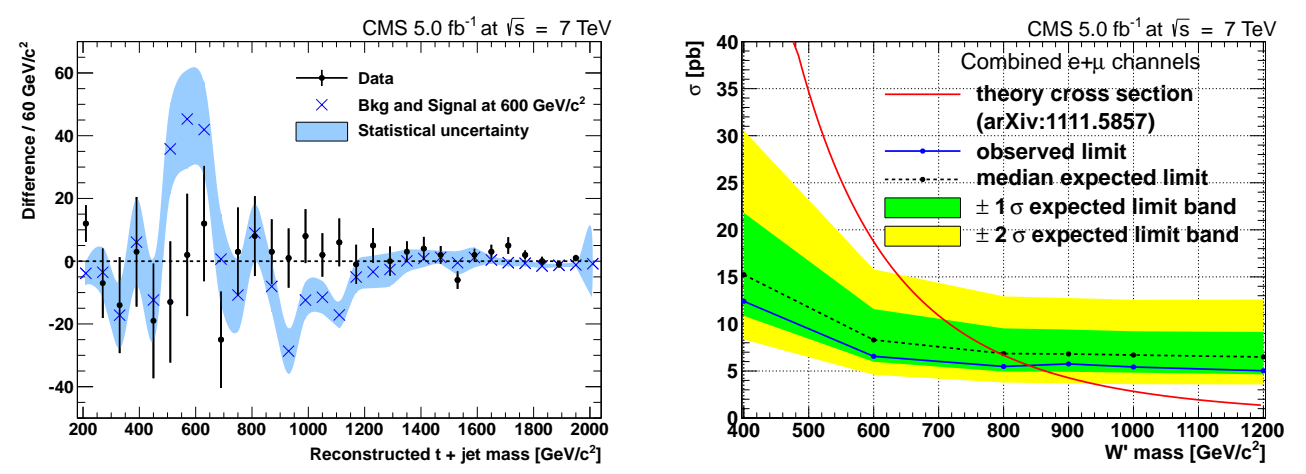

Figure 5: (a) Difference of yields for $t^{-}+d$ and $t^{+}+d$ mass distributions, (b) Production cross section limits as a function of $W^{\prime}$ mass.

\section{4. $W^{\prime}$ or $G_{R S}$ decaying to vector bosons}

Searches for new heavy resonances decaying to $Z W$ or $Z Z$ have been performed, motivated by testing theories that propose alternatives to the Higgs mechanism. The decay chains $W^{\prime} \rightarrow$ $Z W \rightarrow \ell \ell j j$ and $G_{R S} \rightarrow Z Z \rightarrow \ell \ell j j$ have been selected as benchmark models, where $G_{R S}$ denotes a Randall-Sundrum graviton [13]. The lepton and jet pairs in the final state are boosted in case of heavy objects. Therefore each leptonic leg interferes with the isolation definition of the other leg and has to be excluded from the isolation calculation. Since the hadronically decaying vector 
boson also decays to quarks with small opening angle, they often overlap into a single jet, which provides a useful handle to distinguish the signal from the background. The jet is required to be well separated from the lepton pair. Backgrounds are estimated from data and originate mainly from $W+$ jets, $t \bar{t}, \gamma V+$ jets, $Z / \gamma^{*}+$ jets, $Z Z$, and $V V+$ jets, where $V$ denotes either a $W$ or a $Z$. Fig. 6a shows the invariant dimuon mass distribution calculated from $7 \mathrm{TeV}$ data. In Fig. $6 \mathrm{~b}$ the $95 \%$ confidence level exclusion limits are plotted for the $G_{R S}$ scenario, together with results from other experiments and other CMS results, for different values of the coupling $k / \bar{M}_{P} . k$ is the 5-dimensional curvature and $\bar{M}_{P}$ the Planck mass divided by $\sqrt{8 \pi}$.
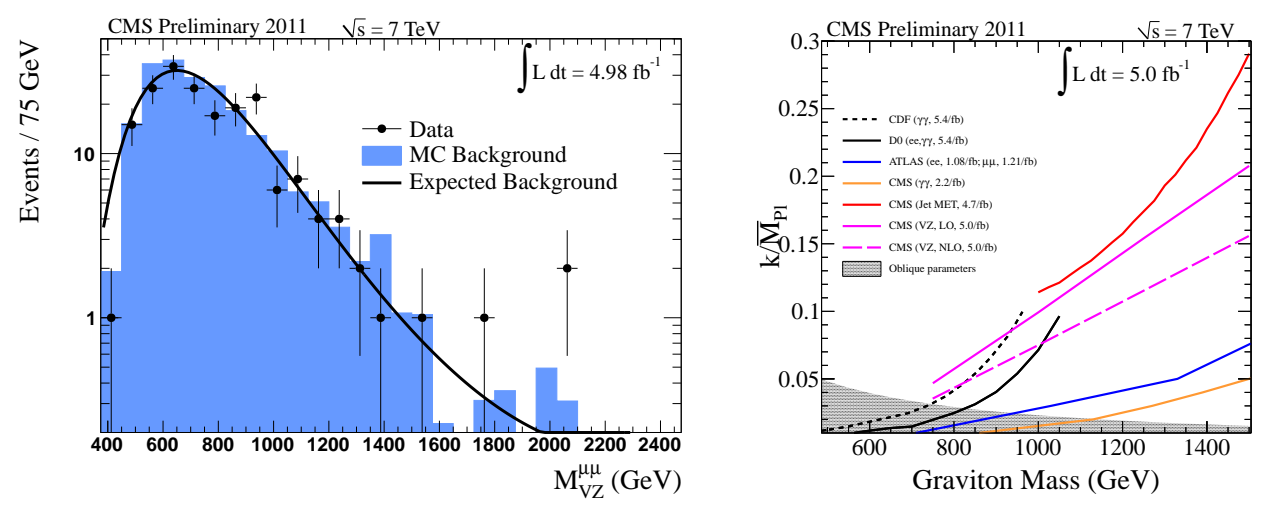

Figure 6: (a) Invariant dimuon mass, (b) Exclusion limits for $G_{R S}$.

\section{References}

[1] G. Altarelli, B. Mele and M. Ruiz-Altaba, Z. Phys. C 45 (1989) 109, [Erratum-ibid. C 47 (1990) 676].

[2] J. C. Pati and A. Salam, Phys. Rev. D 10 (1974) 275, [Erratum-ibid. D 11 (1975) 703].

[3] J. L. Hewett and T. G. Rizzo, Phys. Rept. 183 (1989) 193.

[4] L. Randall and R. Sundrum, Phys. Rev. Lett. 83 (1999) 3370 [hep-ph/9905221].

[5] T. G. Rizzo and J. D. Wells, Phys. Rev. D 61 (2000) 016007 [hep-ph/9906234].

[6] S. Chatrchyan et al. [CMS Collaboration], JINST 3 (2008) S08004.

[7] CMS Collaboration, "Search for Resonances in Dilepton Mass Spectra in pp Collisions at $\sqrt{s}=$ 7 TeV,” CMS Physics Analysis Summary CMS-PAS-EXO-12-015 (2012).

[8] S. Chatrchyan et al. [CMS Collaboration], Phys. Lett. B 716 (2012) 82 [arXiv:1206.1725 [hep-ex]].

[9] R. Diener, S. Godfrey and T. A. W. Martin, Phys. Rev. D 83 (2011) 115008 [arXiv:1006.2845 [hep-ph]].

[10] K. Kong, S. C. Park and T. G. Rizzo, JHEP 1004 (2010) 081 [arXiv:1002.0602 [hep-ph]].

[11] S. Chatrchyan et al. [CMS Collaboration], JHEP 1208 (2012) 023 [arXiv:1204.4764 [hep-ex]].

[12] S. Chatrchyan et al. [CMS Collaboration], Phys. Lett. B 717 (2012) 351 [arXiv:1206.3921 [hep-ex]].

[13] CMS Collaboration, "Search for exotic resonances decaying into $V+Z$ using final states with a jet and a lepton pair," CMS Physics Analysis Summary CMS-PAS-EXO-11-081 (2012). 